\title{
Nivel de autoestima en los adultos mayores de dos grupos de un municipio de Hidalgo, México.
}

\author{
Self-esteem Level in Older Adults of Two Groups in a Municipality of Hidalgo, Mexico. \\ Laura P. Mendoza-Mendoza ${ }^{a}$, Ana M. Martínez-Pérez ${ }^{b}$, Jazmín Rodríguez-Domínguez $^{c}$, \\ Gabriela Maldonado-Muñiz ${ }^{d}$,Claudia A. Trejo-García ${ }^{e}$
}

\begin{abstract}
:
Objective: to identify the level of self-esteem in older adults of two groups. Methodology: quantitative, descriptive, cross-sectional study, conducted in June-August 2018, in the municipality of Ajacuba, Hidalgo, with a sample of 60 older adults, 30 captured in the community (community group) and 30 enrolled in a program of House by day (Group day house), sampling by availability. The Rossemberg self-esteem scale $(\alpha$-Cronbach $=0.79)$ and a sociodemographic data questionnaire were applied through an interview. Results: Community group: 18 were men, 18 were married, 27 did not work, 9 lived with diabetes, 16 with hypertension and 5 without diseases. Day house group: 15 were men, 17 were married, none worked, 20 older adults lived in their own home; 2 smoked tobacco, 3 used medication to sleep, 4 lived with diabetes, 16 with hypertension and 10 without diseases. Community group, 8 with high selfesteem, 17 medium and 5 low; Day house group, 16 with high self-esteem, 12 medium and 2 low. Conclusions: The self-esteem of the older adult of the community was found at the middle level and of those who attend the day house at an elevated level.
\end{abstract}

Keywords:

Elderly, Self-esteem, Community, Day House.

\section{Resumen:}

Objetivo: identificar el nivel de autoestima en los adultos mayores de dos grupos. Metodología: estudio cuantitativo, descriptivo, transversal, realizado en junio-agosto de 2018, en el municipio de Ajacuba, Hidalgo, con una muestra de 60 adultos mayores, 30 captados en la comunidad (Grupo comunidad) y 30 enlistados en un programa de Casa de día (Grupo casa de día), muestreo por disponibilidad. Mediante entrevista se aplicó la escala de autoestima de Rossemberg ( $\alpha$-Cronbach=0.79) y un cuestionario de datos sociodemográficos. Resultados: grupo Comunidad: 18 eran hombres, 18 estaban casados, 27 no laboraban, 9 vivían con diabetes, 16 con hipertensión y 5 sin enfermedades. Grupo casa de día: 15 eran hombres, 17 estaban casados, ninguno laboraba, 20 adultos mayores vivían en casa propia; 2 fumaban tabaco, 3 consumían medicamentos para conciliar el sueño, 4 vivían con diabetes, 16 con hipertensión y 10 sin enfermedades. Grupo comunidad, 8 con autoestima elevada, 17 media y 5 baja; Grupo casa de día, 16 con autoestima elevada, 12 media y 2 baja. Conclusiones: El autoestima del adulto mayor de la comunidad se encontró en nivel medio y de los que asisten a la casa de día en nivel elevado.

\section{Palabras Clave:}

Adulto mayor, Autoestima, Comunidad, Casa de Día.

\footnotetext{
a Alumno de la Universidad Autónoma del Estado de Hidalgo, Escuela Superior de Tlahuelilpan, E-mail: me338661@uaeh.edu.mx

b Alumno de la Universidad Autónoma del Estado de Hidalgo, Escuela Superior de Tlahuelilpan, E-mail: ma338356@uaeh.edu.mx c Alumno de la Universidad Autónoma del Estado de Hidalgo, Escuela Superior de Tlahuelilpan, E-mail: ro340036@uaeh.edu.mx Profesor Investigador de la Universidad Autónoma del Estado de Hidalgo, Escuela Superior de Tlahuelilpan, ORCID: https://orcid.org/0000-0002-4967-1812,Email: gmaldonado@uaeh,edu.mx

e Autor de Correspondencia, Profesor Investigador de la Universidad Autónoma del Estado de Hidalgo, Escuela Superior de Tlahuelilpan, ORCID: https://orcid.org/0000-0003-2205-7879, E-mail: ctrejo@uaeh.edu.mx
} 


\section{Introducción}

La población del mundo envejece rápidamente y ello es motivo de celebración. Por una parte, este fenómeno evidencia que hemos logrado adelantos contra las enfermedades. Sin embargo, el envejecimiento plantea a la vez problemas y oportunidades (OMS, 2015), por lo que es necesario considerar los cambios biológicos, psicológicos y sociales. El objetivo del presente documento fue identificar el nivel de autoestima en los adultos mayores de dos grupos.

\section{Adulto mayor}

Las proyecciones indican que la población mundial está envejeciendo rápidamente, entre 2015 y 2050, pasará de 900 millones hasta 2000 millones de personas con más de 60 años de edad, lo que representa un aumento del $12 \%$ al 22\% (OMS, 2015). La población de adultos mayores representa $10.1 \%$ del total de la población rural y $8.6 \%$ de la población urbana. Las personas adultas mayores residen en su mayoría en hogares familiares: la mitad de los hombres (49.7\%) en nucleares; $38.1 \%$ en ampliados y $1.7 \%$ en compuestos. $37.9 \%$ de las mujeres viven en nucleares y $47 \%$ en hogares ampliados; su presencia en hogares compuestos es similar a la de los hombres adultos mayores. Sin embargo, $12 \%$ de las mujeres y 9.2\% de los hombres adultos mayores viven solos (INAPAM, 2015).

La salud mental y el bienestar emocional tienen la misma importancia en la edad mayor que en cualquier otro periodo de la vida (OMS, 2017). Para el bienestar físico y emocional de los adultos mayores el que se les provea de un ambiente que favorezca su autoestima, donde se promueva su independencia y autonomía y se fomenten las relaciones sociales con otras personas con las que puedan compartir intereses, recuerdos, diversión y, principalmente, donde se valore la experiencia y sabiduría que han adquirido a través de su vida (Gilabert, 2015).

\section{Autoestima}

Rosenberg en 1965 conceptualizó la autoestima como un sentimiento hacia uno mismo, que puede ser positivo 0 negativo, que se construye por medio de una evaluación de las propias características (de León-Ricardi, \& GarcíaMéndez, 2016).

La construcción del concepto de autoestima se forma de la interacción de tres esferas principales: la primera es el Yo (mi manera de actuar, mi manera de ser más conocida); la segunda es lo que influye en mi mundo cercano (mi familia, mis amigos, mis relaciones sociales); y la tercera esfera es la interacción cultural y los conceptos que existen en ella. Por ejemplo, en una sociedad donde el Adulto Mayor es valorado culturalmente y es apreciado por sus conocimientos y sabiduría, es menos probable que se presenten graves problemas de la autoestima, como el constante deseo de morir, ya que esta percepción social ayudará a que el adulto mayor tenga un lugar especial en ese grupo en particular. Por el contrario, en las sociedades modernas el adulto mayor tiene dificultad de conocerse y valorarse, ya que el reconocerse viejo es uno de los valores más criticados y por lo mismo es excluido. La autoestima dará la pauta a conductas en el andar cotidiano.

La autoestima en el adulto mayor puede ser alta o normal cuando la persona se reconoce como importante para sí y los demás, tiene deseos propios, los expresa y defiende; cuando enfrenta las crisis, los cambios y las pérdidas, cuando reflexiona, busca apoyo e información y cuando busca autonomía y la disfruta. Pero también puede presentase una autoestima baja o negativa, como cuando el adulto mayor tiene una poca aceptación de sí mismo, cuando cree que por su edad no sirve o es un estorbo, cuando no acepta los cambios en su cuerpo y se deprime, cuando es negativo, cascarrabias, pesimista y le cuesta dar y recibir afecto (2016)

\section{Planteamiento del problema o exposición del caso}

Una baja autoestima repercute negativamente en todos los ámbitos de la vida. La percepción que tienen los adultos mayores de sí mismos y de su valía o carencia de ella, va a influir irremediablemente en las relaciones personales y en la relación con la sociedad. Cuando se tiene baja autoestima es común sentir una gama de sentimientos negativos como: miedo vergüenza, abandono, rabia, resentimiento, desconfianza, falta de respeto, falta de valía, falta de poder, soledad, aislamiento y pérdida del respeto por sí mismos.

Aplicar la escala de Rosemberg en estudios de valoración de autoestima en adultos mayores podría permitirnos aclarar algunas de las diferencias existentes entre los hallazgos de estudios referentes al nivel de autoestima de dos grupos de la población en un municipio del estado de Hidalgo. Por lo que se planteó la siguiente pregunta de investigación: ¿Cuál es el nivel de autoestima de los adultos mayores de dos grupos?

\section{Conocimiento previo}

En el trabajo titulado Nivel de autoestima de las personas adultas mayores que acuden a los comedores de la Sociedad Pública de Beneficencia de Lima Metropolitana 2011, publicaron que la mayoría (62\%) de los adultos mayores mostraron nivel de autoestima bajo, 18\% 
intermedio y $20 \%$ alto (Villavicencio-Ramírez, \& RiveroÁlvarez, 2014).

\section{Metodología de diseño o de estudio}

Estudio cuantitativo, descriptivo, aplicado y transversal, observacional. La recolección de datos se realizó en el periodo junio - agosto 2018, en el municipio de Ajacuba, Hidalgo, México; muestra de 60 adultos mayores, de los cuales 30 enlistados en un programa de Casa de día (Grupo Casa de día) y 30 que no participan en algún programa social (Grupo Comunidad), muestreo por disponibilidad. Se incluyeron personas mayores de 60 años, que residan en el municipio de Ajacuba y que aceptaron participar, se firmó consentimiento informado.

Se aplicó la Escala de autoestima de Rosemberg, que tiene por objeto evaluar el sentimiento de satisfacción que la persona tiene de sí misma. La escala ha sido traducida y validada en castellano. Validada para población Mexicana. La consistencia interna de la escala se encuentra entre 0.76 y 0.87 . La fiabilidad es de 0.80 ; consta de 10 ítems, frases de las que cinco están enunciadas de forma positiva y cinco de forma negativa, las respuestas se puntúan de 1 a 4 , se interpreta como estima elevada si obtiene de 30 a 40 puntos, considerando una autoestima normal, autoestima media de 26 a 29 puntos, no presenta problemas de autoestima graves, pero es conveniente mejorarla, y autoestima baja si obtiene menos de 25 puntos, mostrando problemas significativos de autoestima (Jurado-Cárdenas, et al., 2015; De León Ricardi, et al., 2016).

\section{V.Aplicación}

La diferencia entre dos grupos de una comunidad, nos da la evidencia para sugerir a los adultos mayores la participación en las actividades de un programa elaborado exprofeso implantado en la Casa de día, donde tiene acceso a orientación para la salud, programas sociales, actividades recreativas y artísticas, con la finalidad de aumentar el autoestima y con ello mejorar la percepción de la calidad de vida de la población de un municipio del estado de Hidalgo, ubicado dentro de las diez entidades con mayor pobreza en el país (CONEVAL, 2012).

\section{Resultados}

La muestra estuvo conformada por 60 adultos mayores, 30 del grupo casa de día de Santiago Tezontlale y 30 del grupo comunidad. Ambos del municipio de Ajacuba, Hidalgo, México.
Grupo Comunidad: $\mathrm{n}=30$ adultos mayors, 18 eran de género masculino y 12 femenino; 18 estaban casados y 12 viudos, 4 nacieron en Santiago Tezontlale y 26 en Ajacuba, 2 tenían un trabajo estable, 1 se dedicaba al hogar y 27 no realizaban actividades de tipo laboral, 7 adultos mayores tenían entre 1-3 hijos, 18 entre 3-6 hijos y 5 más de 6 hijos, 19 vivían en casa propia, los adultos mayores referían que entre las actividades no aprobadas por la sociedad una persona ingería alcohol y una consumía medicamentos para conciliar el sueño, 9 vivían con diabetes, 16 con hipertensión y 5 no padecían ninguna enfermedad.

Grupo Casa de día: 15 eran de género masculino y 15 del género femenino, 17 eran casados, 12 personas eran viudos y uno era divorciado, ninguno realzaban actividades de tipo laboral, 2 adultos mayores tenían entre 1-3 hijos, 15 adultos tenían entre 3-6 hijos y 13 tenían más de 6 hijos, 20 adultos mayores vivían en casa propia y 10 no contaban con casa propia, los adultos mayores referían que entre sus actividades no aprobadas por la sociedad solo 2 fumaban tabaco, 3 consumían medicamentos para conciliar el sueño, 4 vivían con diabetes, 16 con hipertensión y 10 no padecían ninguna enfermedad. Ver tabla 1

Tabla 1. Frecuencia de variables sociodemográficas de acuerdo a grupos, de los adultos mayores de Ajacuba, Hidalgo, México, 2018.

$n=60$

\begin{tabular}{|c|c|c|c|}
\hline \multicolumn{2}{|c|}{$\begin{array}{l}\text { Variables } \\
\text { sociodemográficas }\end{array}$} & \multicolumn{2}{|c|}{ Grupos } \\
\hline & & $\begin{array}{c}\text { Comunidad } \\
\mathrm{f}\end{array}$ & $\begin{array}{c}\text { Casa } \\
\text { de } \\
\text { día } \\
\text { f }\end{array}$ \\
\hline \multirow[t]{2}{*}{ Género } & Masculino & 18 & 15 \\
\hline & Femenino & 12 & 15 \\
\hline \multirow[t]{3}{*}{ Estado civil } & Casado & 18 & 17 \\
\hline & Viudo & 12 & 12 \\
\hline & Divorciado & 0 & 1 \\
\hline \multirow[t]{3}{*}{ Ocupación } & Trabajo & 2 & 0 \\
\hline & Hogar & 1 & 0 \\
\hline & Nada & 27 & 30 \\
\hline \multirow[t]{3}{*}{ Hijos } & $1-3$ & 7 & 2 \\
\hline & $3-6$ & 18 & 15 \\
\hline & Más de 6 & 5 & 13 \\
\hline \multirow[t]{2}{*}{ Casa propia } & Sí & 19 & 20 \\
\hline & No & 11 & 10 \\
\hline \multirow[t]{5}{*}{ Actividades } & Alcohol & 1 & 0 \\
\hline & Tabaco & 0 & 2 \\
\hline & $\begin{array}{l}\text { Medica- } \\
\text { mentos }\end{array}$ & 1 & 3 \\
\hline & Ninguna & 28 & 25 \\
\hline & Diabetes & 9 & 4 \\
\hline
\end{tabular}




\begin{tabular}{llcc}
\hline $\begin{array}{l}\text { Enfermeda- } \\
\text { des }\end{array}$ & $\begin{array}{l}\text { Hiperten- } \\
\text { sión }\end{array}$ & 16 & 16 \\
\cline { 2 - 4 } & Ninguna & 5 & 10 \\
\hline
\end{tabular}

Fuente: Cuestionario de datos sociodemográficos.

Nivel de autoestima:

Grupo Comunidad: $\mathrm{n}=30$ adultos mayores, 8 alcanzaron puntuación elevada, 17 puntuación media y 5 baja.

Grupo Casa de día: $\mathrm{n}=30$ adultos mayores, 16 adquirieron puntuación elevada, 12 puntuación media y 2 baja. Ver tabla 2.

Tabla 2. Frecuencia del nivel de autoestima de acuerdo a grupos, de los adultos mayores de Ajacuba, Hidalgo, México, 2018. $n=60$

\begin{tabular}{|c|c|c|c|c|}
\hline \multirow[t]{2}{*}{ Grupos } & \multicolumn{3}{|c|}{ Nivel de Autoestima } & \multirow[t]{2}{*}{ Total } \\
\hline & $\begin{array}{c}\text { Elevada } \\
f\end{array}$ & $\underset{f}{\text { Media }}$ & $\begin{array}{c}\text { Baja } \\
f\end{array}$ & \\
\hline Comunidad & 8 & 17 & 5 & 30 \\
\hline Casa de día & 16 & 12 & 2 & 30 \\
\hline Total & 24 & 29 & 7 & 60 \\
\hline
\end{tabular}

Fuente: Escala de Rosemberg

\section{Discusión}

De León Ricardi, en el trabajo titulado Escala de Rosemberg en población de adultos mayores realizado en la Ciudad de México en el 2017, encontró $26.18 \%$ de nivel bajo de autoestima (de León-Ricardi, \& García-Méndez, 2016), superior a lo encontrado en los adultos mayores del municipio de Ajacuba con una prevalencia de 11.6\%; la diferencia puede ser por la zona de residencia, ya que las relaciones recíprocas son importantes para la autoestima del adulto mayor (OMS, 2015b), considerando que los participantes nacieron y residen en esta comunidad, las relaciones son fuertes y existe identidad.

\section{Conclusiones}

La autoestima del adulto mayor del grupo comunidad se encuentra en nivel medio y del grupo casa de día en nivel elevado.

Por lo que se recomeinda la integración de los adultos mayores a un programa social en una casa de día.

\section{Agradecimientos}

A las autoridades de la Casa de día de Santiago Tezontlale y al DIF municipal de Ajacuba, Hidalgo, por su apoyo, así como a los adultos mayores por su contribución.

\section{Referencias}

Asociación Mexicana de Gerontología y Geriatría. (2016). La importancia de la autoestima en el Adulto Mayor. Recuperado el 28 de abril de 2019, de Asociación Mexicana de Gerontología y Geriatría A.C.: https://amgg.com.mx/sobre-el-envejecimiento/laimportancia-de-la-autoestima-en-el-adulto-mayor/

CONEVAL. (2012). Informe de pobreza y evaluación en el estado de Hidalgo 2012. Recuperado el 28 de abril de 2019, de Consejo Nacional de Evaluación de la Política de Desarrollo Social:

https://www.coneval.org.mx/coordinacion/entidades/Doc uments/Informes \%20de\%20pobreza\%20y\%20evaluaci\% C3\%B3n\%202010-

2012_Documentos/Informe\%20de\%20pobreza\%20y\%20 evaluaci\%C3\%B3n\%202012_Hidalgo.pdf

De León Ricardi, C.A., \& García Méndez, M. (2016). Escala de Rosenberg en población de adultos mayores. Ciencias Psicológicas, 10(2), 119-127. Recuperado en 28 de abril de 2019, de

http://www.scielo.edu.uy/scielo.php?script=sci_arttext\&p $\mathrm{id}=$ S1688-42212016000200002\&lng=es\&tlng=es.

Gilabert, J. (2015). Importancia de la socialización en el Adulto Mayor. Recuperado el 28 de abril de 2019, de Gericare: http://www.gericare.com.mx/blog/importanciade-la-socializacion-en-el-adulto-mayor/

INAPAM (2015). Situación de las personas adultas mayores en México. Recuperado en 28 de abril de 2019, de Instituto Nacional de las Personas Adultas Mayores: http://cedoc.inmujeres.gob.mx/documentos_download/10 1243.pdf

Jurado-Cárdenas, D., Jurado-Cárdenas, S., López-Villagrán, K., \& Querevalú-Guzmán, B. (2015). Validez de la Escala de Autoestima de Rosenberg en universitarios de la Ciudad de México. Revista Latinoamericana de Medicina Conductual, 5(1), 18-22, Recuperado en 28 de abril de 2019, de http://www.redalyc.org/pdf/2830/283046027004.pdf

OMS (2015). Envejecimiento y ciclo de vida. Recuperado el 2 de julio de 2018, de Organización Mundial de la Salud: http://www.who.int/features/factfiles/ageing/ageing_facts les/

OMS (2015b). Informe Mundial sobre el envejecimiento y la salud en Organización Mundial de la Salud. Recuperado el 28 de abril de 2019, de https://apps.who.int/iris/bitstream/handle/10665/186471/ WHO_FWC_ALC_15.01_spa.pdf?sequence $=1$ 
OMS (2017). La salud mental y los adultos mayores en

Organización Mundial de la Salud. Recuperado el 28 de abril de 2019, de https://www.who.int/es/news-

room/fact-sheets/detail/la-salud-mental-y-los-adultosmayores

Villavicencio-Ramírez, I.J. \& Rivero-Álvarez, R.P. (2014).

Nivel de autoestima de las personas adultas mayores que acuden a los comedores de la Sociedad Pública de Beneficencia de Lima Metropolitana 2011. Revista Científica Alas Peruanas, 1(1), 1-12. Recuperado en 27 de abril de 2019, de

http://dx.doi.org/10.21503/sd.v1i1.422 